\title{
CFD-Simulations of a $4 \pi$-continuous-mode dilution refrigerator for the CB-ELSA experiment
}

\author{
M. Bornstein, H. Dutz, S. Goertz, S. Reeve, S. Runkel* \\ University of Bonn, Germany \\ E-mail: runkel@physik.uni-bonn.de
}

\begin{abstract}
The polarized target group at Bonn operates a dilution refrigerator for double polarization experiments at the Crystal Barrel in Bonn. To achieve high target polarizations and long relaxation times low temperatures are indispensable. To reach temperatures below $30 \mathrm{mK}$ and to allow for the use of an internal polarization magnet, the polarized target group is building a new continuous mode dilution refrigerator. As a optimizing tool for the construction of dilution refrigerators and for a better understanding of the different incoming and outgoing fluid streams several CFDsimulations are done. First the streams are simulated independently for different parts of the refrigerator to get a better estimation of the flow parameters. Then the simulation is extended to include the heat exchange between the different streams at the heat exchangers for different operational parameters of the refrigerator. Afterwards the precooling stages of the refrigerator will be tested to compare the predicted and the measured operational parameters.
\end{abstract}

XVIth International Workshop in Polarized Sources, Targets, and Polarimetry, PSTP2015,

14-18 September 2015,

Bochum, Germany

\footnotetext{
* Speaker.
} 


\section{Introduction}

In order to increase the figure of merit for the CB experiment at ELSA in Bonn the polarized target group predicted a solid continuous mode target, where it is possible to polarize the target material during the data taking [1]. To achieve this goal a complete new dilution refrigerator was developed which is under construction. The refrigerator consists of two main components. The dilution unit which is designed to reach temperatures below $30 \mathrm{mK}$ in frozen spin mode - $200 \mathrm{mK}$ in continuous mode - and the precooling unit which has to cool and liquefy the circulating ${ }^{3} \mathrm{He}-{ }^{4} \mathrm{He}$ dilute before it is refilled into the dilution unit. The goal of the CFD-simulations is to estimate the characteristical flow parameter for every in and out going stream and to simulate the heat exchange between them for a better insight to the operational characteristics of this dilution refrigerator. Simulations for the different parts of the precooling stages were performed.

\section{The precooling stages}

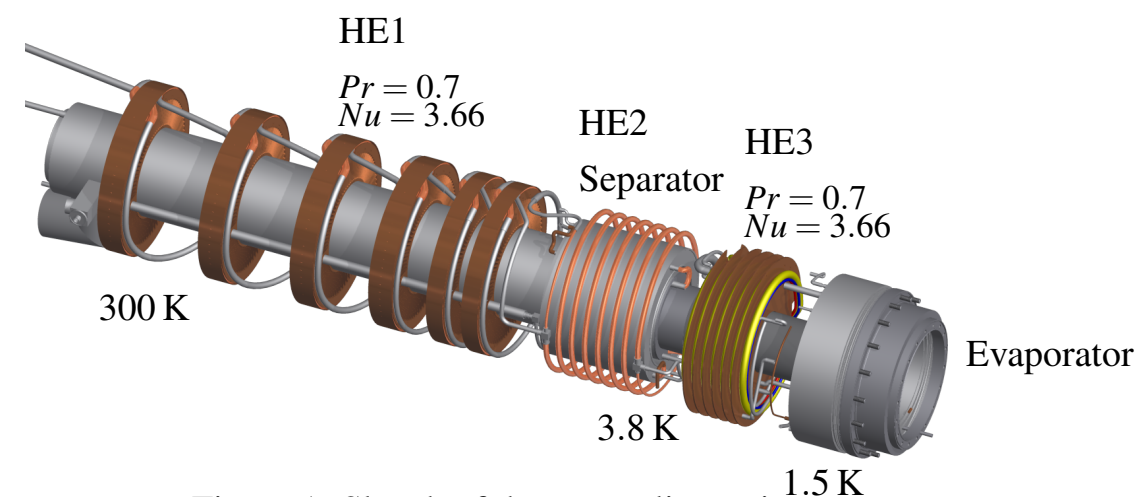

Figure 1: Sketch of the precooling unit

The precooling unit consists of two heat sunks and three heat exchangers which are shown in Fig. 1. One additional heat exchanger is located in the second heat sunk. It is called evaporator and is working at a temperature of $1.5 \mathrm{~K}$. HE1 and HE2 have to precool the incoming dilute by the usage of ${ }^{4} \mathrm{He}$ from the separator and the ${ }^{3} \mathrm{He}$ backstream coming from the still down to the separator temperature of $3.8 \mathrm{~K}$. Fig. 2 shows the necessary ${ }^{4} \mathrm{He}$ flowrate to cool the incoming fluid down to this temperature. HE3 is supported by the liquid ${ }^{4} \mathrm{He}$ of the evaporator and liquefys the incoming helium dilute. The heat exchanger in the evaporator should prevent the incoming stream from being cooled down to $1.5 \mathrm{~K}$ before it enters the dilution unit. Fig. 3 shows a flow scheme for the precooling unit.

The initial calculations for the heat stages of the precooling were done similar to [2] - [7] based on energy conservation for each stream. The main challenge for the calculation of heat exchangers used in horizontal dilution refrigerators is the determination of the heat exchange between the different streams through the solid

$$
\dot{Q}_{\text {solid }}\left(\Delta T_{\mathrm{m}}\right)=\alpha \cdot A \Delta T_{\mathrm{m}} \text { with } \alpha \propto \mathrm{Nu}(\operatorname{Re}, \operatorname{Pr}) \cdot \frac{\lambda}{L} .
$$

$\mathrm{Nu}$ is the NUSSELT number, which depends on Re the REYNOLDS and $\operatorname{Pr}$ the PRANDTL number 


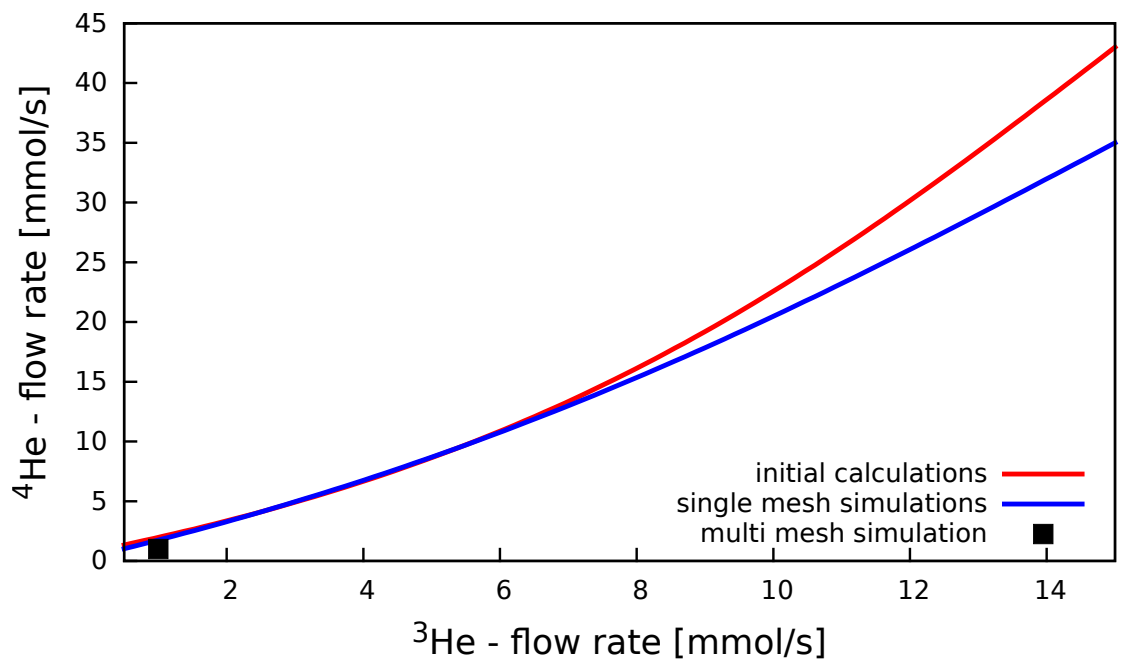

Figure $2:{ }^{4} \mathrm{He}$ flowrate nessesary to cool the incoming dilute to the temperature of the separator

[5]. This three values are used to characterize the stream behaviour. Equation 2.1 shows that the heat exchange depends on the characteristical flow parameter and the geometry. Unfortunately it can be a great challenge to determine this numbers. For the initial calculations the PRANDLT and the REYNOLDS numbers for the ${ }^{3} \mathrm{He}$ backstream were estimated to $\mathrm{Pr}=0.7$ and $\mathrm{Nu}=3.66$. For the other streams the fluid flows through tubes and the calculations from [2] were used, for the calculation of Re and Pr see [4].

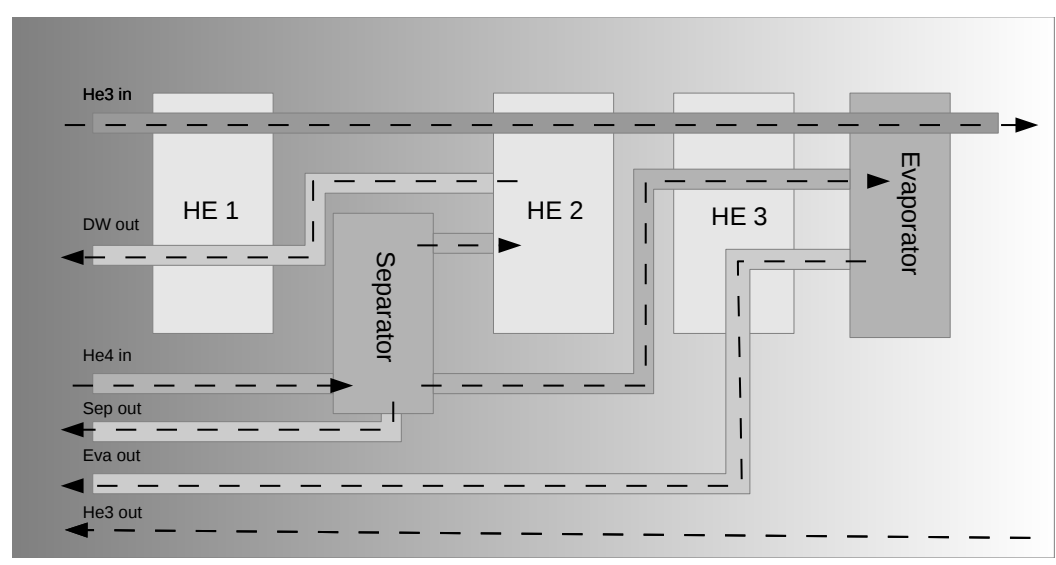

Figure 3: Scheme of the precooling unit and the different streams

\section{CFD-Simulations}

CFD simulations were performed to estimate the influence of the ${ }^{3} \mathrm{He}-{ }^{4} \mathrm{He}$ backstream to the cooling power of the precooling stages. Almost all CFD simulations are based on

$$
\frac{\partial}{\partial t}(\rho \phi)+\underbrace{\nabla \cdot(\rho \underline{u} \phi)}_{F_{\phi}}=D_{\phi}+Q_{\phi} .
$$


$\rho$ is the density, $\phi$ a fluid dynamic parameter (e.g. fluid velocity $\underline{u}$, pressure $p$ or temperature $T$...), $F_{\phi}$ and $D_{\phi}$ are the convective and the diffusive flow and $Q_{\phi}$ represents all other distributions given by $\phi$. For a compressible fluid this leads to

$$
\begin{aligned}
\frac{\partial}{\partial t}(\rho)+\nabla \cdot(\rho \underline{u}) & =0, \\
\frac{\partial}{\partial t}(\rho \underline{u})+\nabla \cdot(\rho \underline{u u}) & =\nabla \cdot \underline{\underline{\tau}}-\nabla p+\rho \underline{g}, \\
\frac{\partial}{\partial t}(\rho h)+\nabla \cdot(\rho \underline{u} h) & =-\nabla \cdot q^{\prime \prime}+\frac{\partial p}{\partial t}+\nabla \cdot(\underline{\underline{\tau}} \cdot \underline{u}) .
\end{aligned}
$$

Depending on the specific case additional conditions like the equation of state or different specific material equations lead to a solvable problem. This simulations were performed with OpenFOAM [8]. The meshes for the calculation were created with the including mesh generating code called snappyHexMesh. There are two possible ways of simulation:

1. Single mesh simulations: a simulation of a single stream to get additional information about the characteristical flow parameters. This simulations are based on the solver rhoPorousSimpleFoam [8].

The advantage of this method is that higher mesh resolutions are possible and it is easier to determine the charactersitical flow parameter like the PRANDTL, the NUSSELT and the REYNOLDS number. These values characterizes the thermal coupling between the fluid and the solid in a system and if the flow is laminar or turbulent. The simulation can be performed for all streams until the flow parameter are known. In addition the characteristical flow parameters can be used to perform the calculations from section 2 again.

2. Multi mesh simulations: a simulation with a different submesh for the calculation of the heat exchange between the different fluid streams and solids. This simulations are based on the solver chtMultiRegionSimpleFoam [8].

By the usage of a multi mesh it is possible to set the boundary conditions for each stream in one simulation and run the solvers for each submesh representing a solid or a fluid. In this case the data necessary for the connecting surfaces of the submeshes can be replaced in each iteration and couple the solvers for each region. With this method the heat exchange between the different streams of the heat exchanger can be calculated in one simulation.

For both solvers we added the low temperature data from [10] - [12] to the thermophysical library implemented in these solvers. The viscosity of the helium gas was modulated with the SUTHERLAND equation [5]. The heat capacity $c_{\mathrm{p}}$ was assumed to be constant for the gaseous streams.

\section{Single Stream Simulations}

In the beginning single stream simulations of the ${ }^{3} \mathrm{He}$ backstream were performed due to the lack of knowledge about the flow parameter of the backstream. Figure 4 shows the pressure and temperature gradient and the velocity profile as an example of this simulations. Here the backstream around heat exchanger 1, 2 and the separator is shown. The beamline and the outer wall are expected to be adiabatic. The massflow is set to $1 \mathrm{mmol} \mathrm{s}^{-1}$ and the temperature is set to $3 \mathrm{~K}$ at the 
inlet. The heat transfer through the solids is given by the FOURIER equation. For this simulation a constant mean temperature given by the calculations of section 2 was estimated for each cooling stage. These initial boundary conditions for the temperature are given in figure 4a.

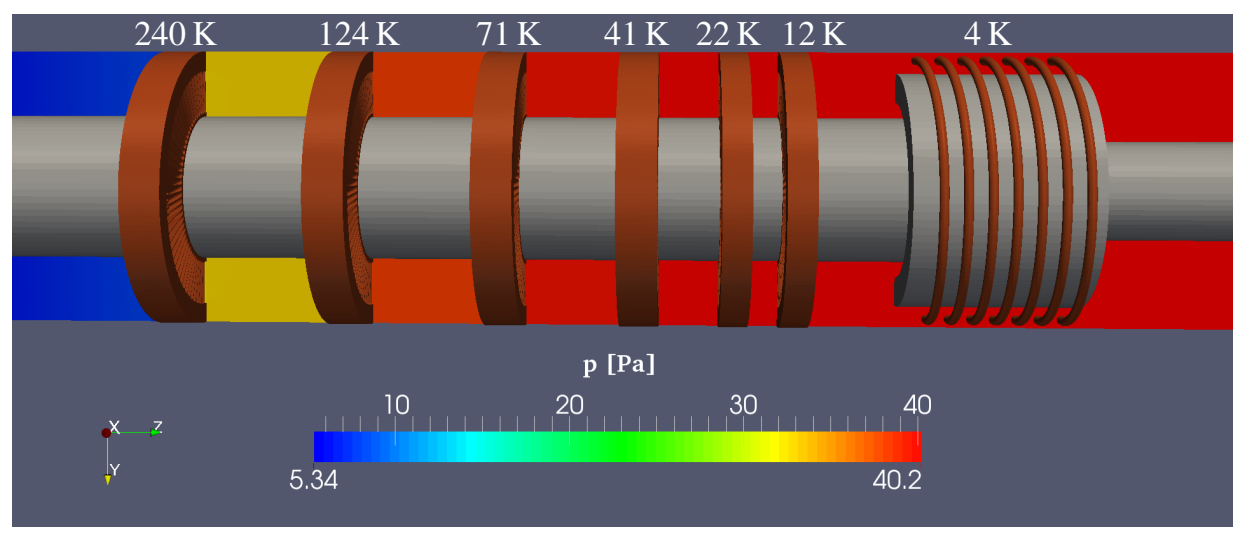

(a) pressure for the ${ }^{3} \mathrm{He}$ backstream

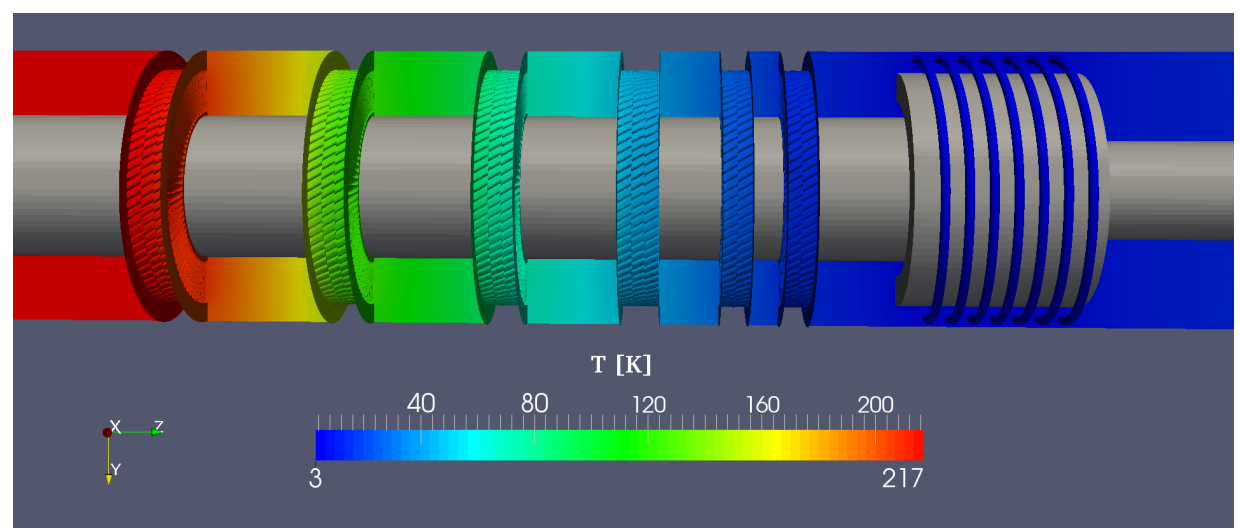

(b) temperature for the ${ }^{3} \mathrm{He}$ backstream

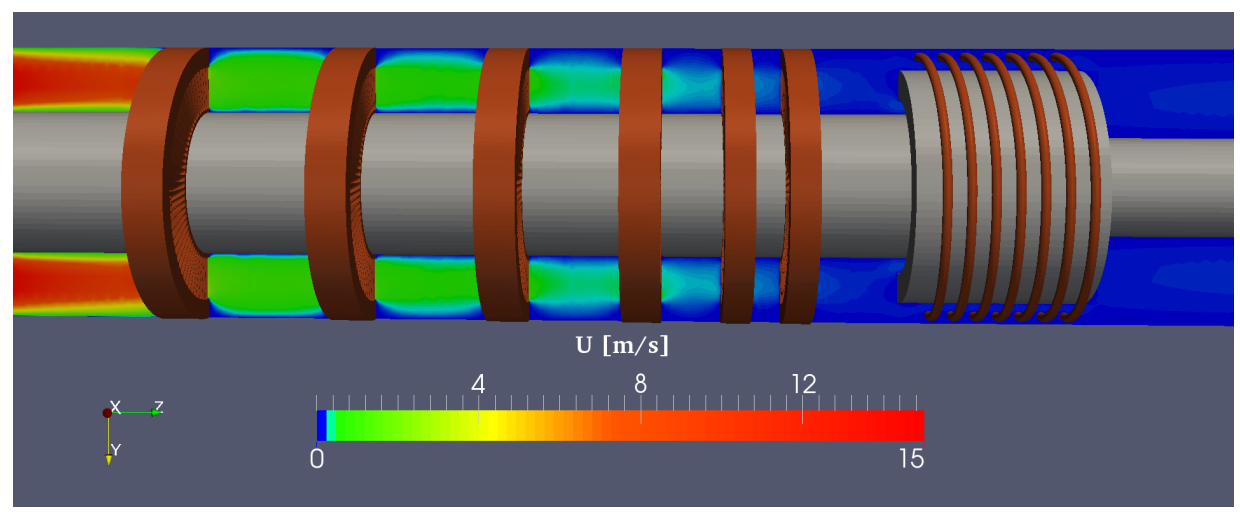

(c) velocity profile for the ${ }^{3} \mathrm{He}$ backstream

Figure 4: Simulation of the ${ }^{3} \mathrm{He}$ backstream for HE1 and HE2 at $1 \mathrm{mmol} \mathrm{s}^{-1}$

The simulations were performed until a residual of $\leq 10 \times 10^{-7}$ for all parameters was reached. Due to the heat exchange between the different streams this single mesh simulations have to be repeated until convergence for the complete system is reached. It is also possible to extract $\mathrm{Re}, \mathrm{Pr}$ 
and $\mathrm{Nu}$ from these data. Afterwards the necessary ${ }^{4} \mathrm{He}$ flow-rate to precool the circulating dilute can be recalculated, this is displayed in Fig. 2 by the blue line.

\section{Simulation of an Heat Exchanger}

Multi region simulations were performed to calculate the heat transfer between the different streams and the important flow parameters of a heat exchanger in one simulation. In Fig. 5a - 5b the pressure gradient for the incoming and outgoing ${ }^{3} \mathrm{He}$ is shown for HE2 as an example of this simulation. The temperature gradient for the different streams is given in Fig. $5 c-5 d$. For the simulation shown in Fig. 5 a ${ }^{3} \mathrm{He}$ circulation rate of $1 \mathrm{mmol} \mathrm{s}^{-1}$ were estimated. The inlet temperatures for the ${ }^{3} \mathrm{He}$ backstream and the ${ }^{4} \mathrm{He}$ are set to $3.8 \mathrm{~K}$, the inlet temperature of the incoming ${ }^{3} \mathrm{He}$ is estimated to $5 \mathrm{~K}$. The simulations for all heat exchangers of the precooling stages with the flow rates for the different operation modes are ongoing. With this data a prediction for the necessary ${ }^{4} \mathrm{He}$ flow rate to precool the circulating dilute under the influence of the ${ }^{3} \mathrm{He}$ back-stream at a given circulating rate can be done. Fig. 2 also shows the prediction from the multi region simulations for the flow parameter of successfully completed simulations at this time.

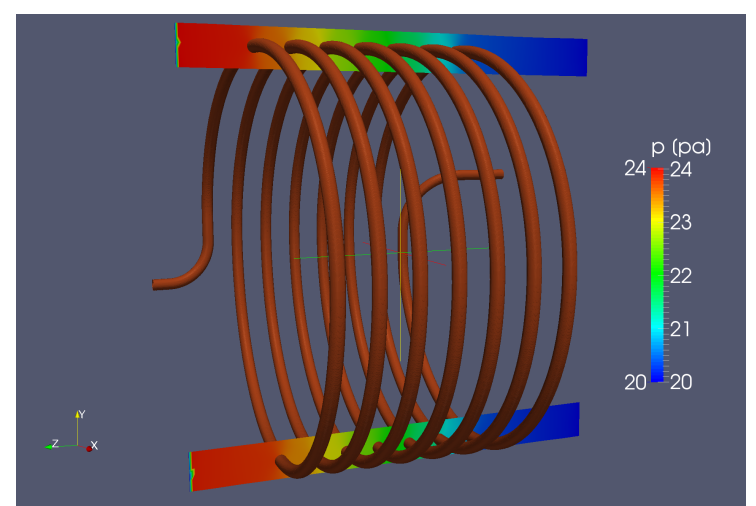

(a) pressure for the ${ }^{3} \mathrm{He}$ backstream

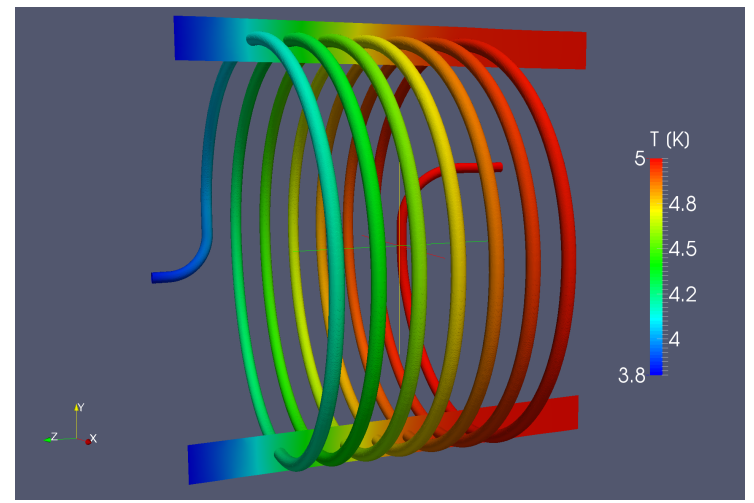

(c) temperature of the incoming and the outgoing ${ }^{3} \mathrm{He}$ stream

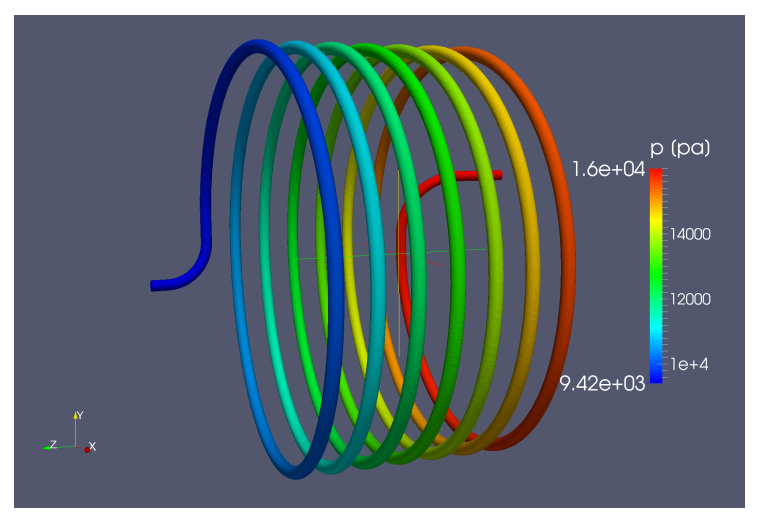

(b) pressure for the incoming ${ }^{3} \mathrm{He}$ stream

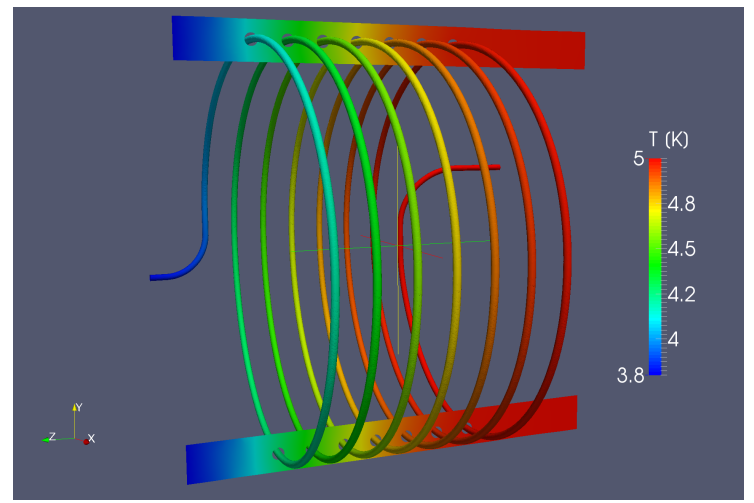

(d) temperature of the outgoing ${ }^{3} \mathrm{He}$ and ${ }^{4} \mathrm{He}$

Figure 5: Multi mesh simulation of HE2 at a ${ }^{3} \mathrm{He}$ circulation rate of $1 \mathrm{mmol} \mathrm{s}^{-1}$

Now it is possible to access the velocity profile, the temperature and the pressure gradient for all 
parts of a heat exchanger within one simulation. This information can also be used to optimize the performance of the different cooling stages by changing the geometry and by optimizing the flow impedances.

\section{Conclusion and Outlook}

The polarized target group in Bonn developes a new horizontal continuous mode target for the $\mathrm{CB}$ experiment at ELSA. To increase the knowledge about the flow characteristics of a horizontal dilution refrigerator CFD simulations were set up. It is possible to use multi region simulations to calculate the performance of the different heat exchangers of a dilution refrigerator (see sec. 5). This simulations are ongoing with the different flow parameter, which can expected for the new continuous mode dilution refrigerator for the CB-ELSA experiment. With this additional information we are able to optimize the concept of our dilution refrigerator which is under construction. After finishing the construction performance tests will be started and the experimental data will be compared with the simulations.

\section{References}

[1] H. Dutz, in proceedings of $16^{\text {th }}$ Int. Spin Phy. Symp. Trieste, Italy 2004, World Scientific (2005) 221

[2] C. Bradke, A New Frozen-Spin Target for the Measurement of the Helicity Asymmetry of the Total Photoabsorption Cross-Section Bonn University, Germany BONN-IR-2000-1

[3] N. Borisov, personal communication

[4] W. Polifke and J.Kopitz, Wärmeübertragung: Grundlagen, analytischer und numerische Methoden Munich, Germany, Pearson Studium, 2nd edition (2009), chapter 8

[5] H. Oertel, Prandtl - Führer durch die Ströhmungslehre Wiesbaden, Germany 2012, Springer

[6] G. Frosatti, Experimental Techniques: Methods for Cooling Below $300 \mathrm{mK}$, Journal of Low Temperature Physics, 1992, Vol. 87, Nos. 3/4

[7] Frank Pobell, Mater and Methods at Low Temperatures Berlin, Germany (2007), Springer 3rd. edition

[8] OpenFOAM Foundation, OpenFOAM User Guide, Version 2.3.0, 5th February 2014

[9] J. P. Van Doormal and G. D. Raithby, Enhancement of the SIMPLE Method for Predicting compressible Flows, Num. Heat Trans 7 147-163

[10] NIST, Cryogenics Technologies Group, Website (2010), http://cryogenics.nist.gov/MPropsMAY/materialproperties.htm

[11] NIST , Chemical Web book, Website (2010), http://webbook.nist.gov/chemistry/fluid/

[12] W. E. Keller, ${ }^{3} h e$ and ${ }^{4} h e, 2$ ed., Plenum Press (1969). 